\title{
The Slow Passage Through the Resonances and Wave Packets with the Different Carriers
}

\author{
Oleg Kiselev and Sergei Glebov \\ Communicated by Y. Charles Li, received May 4, 2005
}

\begin{abstract}
Solution of the nonlinear Klein-Gordon equation perturbed by small external force is investigated. The perturbation is represented by finite collections of harmonics. The frequencies of the perturbation vary slowly and pass through the resonant values consecutively. The resonances lead to the sequence of the wave packets with the different fast oscillated carriers. Full asymptotic description of this process is presented.
\end{abstract}

\section{Contents}

1. Introduction 261

2. Main result 262

3. Pre-resonance expansion 264

4. Internal asymptotics 268

5. Post-resonance expansion 275

6. Resonances in the higher order terms of the asymptotics. 278

References

\section{Introduction}

In this work we study the problem of a generation of sequences of solitary packets with different carriers in the optical fiber. The nonlinear Klein-Gordon equation is studied as a modeling equation. We consider this equation which is perturbed by a small external driving force with finite collection of modes. The external perturbation relates to the pumping of the nonlinear media by an electromagnetic

1991 Mathematics Subject Classification. Primary: 35; Secondary: 78.

Key words and phrases. Klein-Gordon equation, wave packets, finite collections of harmonics, asymptotics, resonance.

This work was supported by grants RFBR 03-01-00716, 04-02-97503, Leading Scientific Schools 1446.2003.1 and INTAS 03-51-4286. 
field. The multiple-frequency pumping allows to increase the capacity of the optical communication channel. The wave packets appear due to passage through a resonance by different modes of the external force. After the passage through the whole resonances the solution contains the full collection of the solitary packages of waves with different carriers. The envelope functions of these packages satisfy to nonlinear Schrödinger equation (NLSE).

In general the derivation of NLSE for small solutions of nonlinear equations is well known [1, 2, 3] and justified [4. Our solution has a more complicate structure. Before all resonances the solution has an order of the perturbation and defined by the external force. After the passage through the resonance of the last mode of the perturbation the solution has the order of the square root of the order of the perturbation and satisfies NLSE. So we show the process of the resonant transformation of the solution and the appearance of the wave packets with the different carriers.

Earlier the resonant generation of periodic waves by a small external force was investigated by a computer simulation $\mathbf{5}$. The phenomenon of the generation and the scattering of the solitary waves in the case of nonlinear Schrodinger equation was asymptotically investigated in $\mathbf{6}, \mathbf{7}$. The problem on the generation of a solitary wave with a single carrier was solved in $\mathbf{8}, \mathbf{9}$. Here we use the proposed approach to study the multiphase case.

The proposed approach is based on a local resonance phenomenon. The local resonance in linear ordinary differential equations was investigated in papers [10, 11. Later this phenomenon was investigated in partial differential equations for linear case [12] and for weak nonlinear case [13, 14.

The goal of this paper is the following: to demonstrate that the passage through the resonances allows to obtain the sequences of solitary packets of waves with the different carriers. The packets with different carriers do not interact.

This paper has the following structure. The first section contains the statement of the problem, main result and example. The second section contains the asymptotic construction before the resonance. In the third section we construct an internal asymptotics in the neighborhood of the resonance curve. In the fourth section we construct the asymptotic expansion after the passage through the resonance. Five section contains the analysis of the resonances in high-order terms of the asymptotic solution. All asymptotics are matched.

\section{Main result}

Let us consider the Klein-Gordon equation with the cubic nonlinearity perturbed by external force with the finite collection of the different harmonics

$$
\partial_{t}^{2} U-\partial_{x}^{2} U+U+\gamma U^{3}=\varepsilon^{2} \sum_{k=1}^{N} f_{k}(\varepsilon x) \exp \left\{i k \frac{S\left(\varepsilon^{2} t, \varepsilon^{2} x\right)}{\varepsilon^{2}}\right\}+\text { c.c. }, \quad 0<\varepsilon \ll 1 .
$$

Here $\gamma=$ const; $f_{k}(y), k=1, \ldots, N$ and the phase function $S(y, z)$ are smooth.

We construct the formal asymptotic solution of the WKB-type. In the domain when the first mode of the perturbation does not pass through the resonance the 
asymptotic solution has a form

$$
U=-\varepsilon^{2} \sum_{j=1}^{N} \frac{f_{j}}{l_{j}} \exp \left\{i(j S) / \varepsilon^{2}\right\}+O\left(\varepsilon^{3}\right),
$$

where

$$
l_{j}\left(x_{2}, t_{2}\right) \equiv j^{2}\left(\partial_{t_{2}} S\right)^{2}-j^{2}\left(\partial_{x_{2}} S\right)^{2}-1, \quad x_{2}=\varepsilon^{2} x, t_{2}=\varepsilon^{2} t .
$$

The leading-order term has an order of $\varepsilon^{2}$ and oscillates. Such solution relates to the forced oscillations.

On the curve $l_{k}\left(x_{2}, t_{2}\right)=0, k=N, \ldots, 1$ the frequencies of $k$-th mode of the forced oscillations and a frequency of the eigen oscillations of the linearized Klein-Gordon equation are equal. Usually the curves with the such property are called resonant curves. The local resonance layer appears in the neighborhood of the curve $l_{k}\left(x_{2}, t_{2}\right)=0$. Here we describe the passage through the resonant layer with the ordinary number $k$.

After passage through the $k$-th resonant layer a new eigen mode with the amplitude of the order $\varepsilon$ appears in the solution. It leads to changing of the number of harmonics in leading-order term. The amplitudes of the leading-order term satisfy $(N-k+1)$ nonlinear Schrödinger equations after passage through the $k$-th resonance. The solution has a form

$$
U=\varepsilon \sum_{\alpha_{3}} \Psi_{\alpha} \exp \left\{i \alpha_{3} / \varepsilon^{2}\right\}+O\left(\varepsilon^{2}\right),
$$

where $\alpha=\left(1,0, \alpha_{3}\right)$ is a multiindex. The variable $\alpha_{3}$ possesses the value from $\phi_{k}$ up to $\phi_{N}$. The value $k$ in $(2.3)$ is defined by

$$
k=\min j: l_{j}>0, \quad j=1, \ldots, N
$$

and functions $l_{j}\left(x_{2}, t_{2}\right)$ are arrayed:

$$
l_{j}\left(x_{2}, t_{2}\right)<l_{m}\left(x_{2}, t_{2}\right), \quad 1 \leq j<m \leq N .
$$

The accurate formulation of the result for this paper is following

THEOREM 2.1. Let the asymptotic solution of 2.1 relates to forced oscillations:

$$
U=-\varepsilon^{2} \sum_{j=1}^{N} \frac{f_{j}}{l_{j}} \exp \left\{i(j S) / \varepsilon^{2}\right\}+O\left(\varepsilon^{3}\right)
$$

in the domain $l_{N}<0$ before the passage through the first resonance and the asymptotic solution has a form

$$
U=\varepsilon \sum_{\alpha_{3}} \Psi_{\alpha} \exp \left\{i \alpha_{3} / \varepsilon^{2}\right\}+O\left(\varepsilon^{2}\right), \quad \alpha=\left(1,0, \alpha_{3}\right),
$$

in the domain $-l_{k} \gg \varepsilon, \quad l_{k+1} \gg \varepsilon$ before the $k$-th resonance curve $l_{k}=0$. Here

$$
\alpha_{3} \in \Upsilon_{1,0}^{k+1}=\left\{ \pm \phi_{k+1}, \pm \phi_{k+2}, \ldots, \pm \phi_{N}\right\}
$$

and the amplitudes $\Psi_{\alpha}$ satisfies $(N-k)$ nonlinear Schrodinger equations

$$
2 i \partial_{t_{2}} \phi_{j} \partial_{t_{2}} \Psi_{\alpha}+\partial_{\xi_{j}}^{2} \Psi_{\alpha}+i\left[\partial_{t_{2}}^{2} \phi_{j}-\partial_{x_{2}}^{2} \phi_{j}\right] \Psi_{\alpha}+\gamma\left|\Psi_{\alpha}\right|^{2} \Psi_{\alpha}=0,
$$

where $\alpha=\left(1,0, \phi_{j}\right)$ and $j=k+1, \ldots, N$.

Then in the domain $-l_{k-1} \gg \varepsilon, \quad l_{k} \gg \varepsilon$ the solution has the similar structure with the changes of the phase collection according to

$$
\Upsilon_{1,0}^{k}=\Upsilon_{1,0}^{k+1} \cup\left\{ \pm \phi_{k}\right\}
$$


The number of equations in 2.4) increases up to $(N-k+1)$.

Here we use the upper indexes ${ }^{k+1}$ in our notations to specify the number of the resonant curve that was crossed in our constructions.

To illustrate the the main result let us consider equation (2.1) with the simplest driving force with two modes:

$$
F=f_{1}\left(x_{1}\right) \exp \left(i\left(t_{2}^{2} / 2+x_{2}\right) / \varepsilon^{2}\right)+f_{2}\left(x_{1}\right) \exp \left(i\left(t_{2}^{2}+2 x_{2}\right) / \varepsilon^{2}\right) .
$$

In this case the curves of the local resonance are the lines $t_{2}=\sqrt{17 / 16}$ and $t_{2}=\sqrt{2}$. In the domain $t_{2}<\sqrt{17 / 16}$ the asymptotic solution is

$$
U=O\left(\varepsilon^{2}\right) .
$$

The solution of this order with respect to $\varepsilon$ relates to forced oscillations.

After the passage through the first resonance the solution is

$$
U=\varepsilon \Psi_{1,0, \phi_{2}} \exp \left\{i \phi_{2} / \varepsilon^{2}\right\}+O\left(\varepsilon^{2}\right), \quad \sqrt{17 / 16}<t_{2}<\sqrt{2}
$$

The amplitude $\Psi_{1,0, \phi_{2}}$ satisfies the Cauchy problem for

$$
2 i \partial_{t_{2}} \Psi_{1,0, \phi_{2}}+\partial_{\xi_{1} \xi_{1}}^{2} \Psi_{1,0, \phi_{2}}+\gamma\left|\Psi_{1,0, \phi_{2}}\right|^{2} \Psi_{1,0, \phi_{2}}=0
$$

variable $\xi_{1}=t_{1}-\sqrt{17 / 16} x_{1}$, Initial condition is

$$
\left.\Psi_{1,0, \phi_{2}}\right|_{t_{2}=\sqrt{17 / 16}}=f_{1}(\xi)(1+i) \sqrt{\pi} .
$$

After the passage through the second resonance the solution is

$$
U=\varepsilon \Psi_{1,0, \phi_{1}} \exp \left\{i \phi_{1} / \varepsilon^{2}\right\}+\varepsilon \Psi_{1,0, \phi_{2}} \exp \left\{i \phi_{2} / \varepsilon^{2}\right\}+O\left(\varepsilon^{2}\right), \quad t_{2}>\sqrt{2} .
$$

The amplitudes $\Psi_{\left(1,0, \phi_{1}\right)}$ and $\Psi_{\left(1,0, \phi_{2}\right)}$ are determined from the Cauchy problem for two nonlinear Shrödinger equation:

$$
\begin{aligned}
& 2 i \partial_{t_{2}} \Psi_{1,0, \phi_{1}}+\partial_{\xi_{1} \xi_{1}}^{2} \Psi_{1,0, \phi_{1}}+\gamma\left|\Psi_{1,0, \phi_{1}}\right|^{2} \Psi_{1,0, \phi_{1}}=0 \\
& 2 i \partial_{t_{2}} \Psi_{1,0, \phi_{2}}+\partial_{\xi_{2} \xi_{2}}^{2} \Psi_{1,0, \phi_{2}}+\gamma\left|\Psi_{1,0, \phi_{2}}\right|^{2} \Psi_{1,0, \phi_{2}}=0 .
\end{aligned}
$$

Here $\xi_{2}=t_{1}-\sqrt{2} x_{1} / 2$, initial conditions are

$$
\left.\Psi_{1,0, \phi_{2}}\right|_{t_{2}=\sqrt{2}}=\left.\Psi_{1,0, \phi_{2}}\right|_{t_{2}=\sqrt{2}-0},\left.\quad \Psi_{1,0, \phi_{1}}\right|_{t_{2}=\sqrt{2}}=f_{2}\left(\xi_{2}\right)(1+i) \sqrt{\pi} .
$$

The solution of this Cauchy problem contains solitary waves if the initial data are sufficiently large [17.

\section{Pre-resonance expansion}

In this section we construct the formal asymptotic solution in the domain before the $k$-th resonant curve. Here we use scaled variables $x_{j}=\varepsilon^{j} x, t_{j}=\varepsilon^{j} t, j=1,2$. The solution has a form

$$
\begin{gathered}
U(x, t, \varepsilon)=\varepsilon \Psi_{1}^{k+1}+\sum_{n=2}^{\infty} \varepsilon^{n} \Psi_{n}^{k+1}, \\
\Psi_{1}^{k+1}=\sum_{ \pm \phi_{m} \in \Upsilon_{1,0}^{k+1}} \exp \left\{ \pm i \phi_{m}\left(x_{2}, t_{2}\right) / \varepsilon^{2}\right\} \Psi_{1,0, \pm \phi_{m}}\left(x_{1}, t_{1}, t_{2}\right)
\end{gathered}
$$


The higher order terms are

$$
\begin{array}{r}
\Psi_{n}^{k+1}=\sum_{j=0}^{n-2} \ln ^{j}(\varepsilon) \sum_{m=k+1}^{N}\left(\sum_{ \pm \phi_{m}} \exp \left\{ \pm i \phi_{m}\left(x_{2}, t_{2}\right) / \varepsilon^{2}\right\} \Psi_{n, j, \pm \phi_{m}}\left(x_{1}, t_{1}, t_{2}\right)+\right. \\
\left.\sum_{\chi \in \Upsilon^{k+1}{ }_{n, j}^{\prime}} \exp \left\{i \chi\left(x_{2}, t_{2}\right) / \varepsilon^{2}\right\} \Psi_{n, j, \chi}\left(x_{1}, t_{1}, t_{2}\right)\right),
\end{array}
$$

where $\Upsilon_{n, j}^{k+1 \prime}$ is a set of phase functions which is determined by

$$
\begin{aligned}
& \Upsilon_{1,0}^{k+1}=\left\{ \pm \phi_{k+1}, \pm \phi_{k+2}, \ldots, \pm \phi_{N}\right\} ; \quad \Upsilon_{2,0}^{k+1}=\Upsilon_{1,0}^{k+1} \cup\{ \pm S, \ldots, \pm N S\}, \\
& \Upsilon_{n, j}^{k+1}=\bigcup \quad \chi_{n_{1}, j_{1}}+\chi_{n_{2}, j_{2}}+\chi_{n_{3}, j_{3}}, \chi_{n_{p}, j_{p}} \in \Upsilon_{n_{p}, j_{p}}^{k+1} . \\
& n_{1}+n_{2}+n_{3}=n \text {, } \\
& j_{1}+j_{2}+j_{3}=j \\
& \Upsilon_{n, j}^{k+1 \prime}=\Upsilon_{n, j}^{k+1} \backslash \Upsilon_{1,0}^{k+1}
\end{aligned}
$$

This solution contains the two parts. The first part of the solution has the leadingorder term of the order of $\varepsilon$. The phase set of this part is

$$
\Upsilon_{1,0}^{k+1}=\left\{ \pm \phi_{k+1}, \pm \phi_{k+2}, \ldots, \pm \phi_{N}\right\}
$$

. The modes have appeared in the solution due to the crossing of the previous $(N-k)$ resonant curves $l_{j}=0$. The terms of the order of $\varepsilon^{2}$ with the phases $\notin \Upsilon_{1,0}^{k+1}$ relate to the forced oscillations and describe the behaviour of the solution before the first resonance curve $l_{N}=0$ where the forced oscillations take place only.

The asymptotics constructed in this section is valid as $l_{k+1} \gg \varepsilon,-l_{k} \gg \varepsilon$. The result of this section is formulated below.

Note. Expansion (3.1) contains the terms $\varepsilon^{n} \ln ^{m} \varepsilon$. It looks a little bit unexpectedly because of the original equation does not contain the logarithmic terms with respect to $\varepsilon$. But these terms naturally appear due to the passage through the previous resonant layers, see Lemma 4.6 in subsection 4.3.1.

Let us construct the formal asymptotic solution for equation (2.1) in form (3.1). We substitute (3.1) in equation 2.1) and collect the terms of the same order of $\varepsilon$. As a result we obtain a recurrent sequence of equations for the coefficients of the asymptotics.

Terms of the order of $\varepsilon$ give us the equations for the phase functions

$$
\left(\partial_{t_{2}} \phi_{j}\right)^{2}-\left(\partial_{x_{2}} \phi_{j}\right)^{2}-1=0, \quad j=k+1, \ldots, N .
$$

Initial data for the phase functions $\phi_{j}, j=k+2, \ldots, N$ are determined by their values on the curve $l_{k+1}=0$. Initial data for $\phi_{k+1}$ is related with

$$
\left.\phi_{j}\right|_{l_{k+1}=0}=\left.(k+1) S\right|_{l_{k+1}=0},\left.\quad \partial_{t_{2}} \phi_{j}\right|_{l_{k+1}=0}=\left.(k+1) \partial_{t_{2}} S\right|_{l_{k+1}=0} .
$$

The terms of the order of $\varepsilon^{2}$ give us the homogeneous transport equation

$$
\partial_{t_{2}} \phi_{j} \partial_{t_{1}} \Psi_{1,0, \phi_{j}}-\partial_{x_{2}} \phi_{j} \partial_{x_{1}} \Psi_{1,0, \phi_{j}}=0 . \quad j=k+1, \ldots, N .
$$

This equation allows us to determine the dependence of the leading-order term on characteristic variable $\zeta$. Equation (3.4) along the characteristics

$$
\frac{d x_{1}}{d \zeta_{j}}=-\partial_{x_{2}} \phi_{j}, \quad \frac{d t_{1}}{d \zeta_{j}}=\partial_{t_{2}} \phi_{j}
$$


can be written in the form of ordinary differential equation

$$
\frac{d \Psi_{1,0, \phi_{j}}}{d \zeta_{j}}=0
$$

It yields $\Psi_{1,0, \phi_{j}}$ depends on $\xi_{j}$, where the $\xi_{j}$ is defined by

$$
\frac{d x_{1}}{d \xi_{j}}=\partial_{t_{2}} \phi_{j}, \quad \frac{d t_{1}}{d \xi_{j}}=\partial_{x_{2}} \phi_{j} .
$$

Among the terms of the order $\varepsilon^{3}$ we collect the terms which oscillate as $\exp \left(i \phi_{j} / \varepsilon^{2}\right)$. It gives

$$
\begin{array}{r}
2 i\left(\partial_{t_{2}} \phi_{j} \partial_{t_{1}} \Psi_{2,0, \phi_{j}}-\partial_{x_{2}} \phi_{j} \partial_{x_{1}} \Psi_{2,0, \phi_{j}}\right) \\
+2 i \partial_{t_{2}} \phi_{j} \partial_{t_{2}} \Psi_{1,0, \phi_{j}}+\left[\left(\partial_{t_{1}} \xi_{j}\right)^{2}-\left(\partial_{x_{1}} \xi_{j}\right)^{2}\right] \partial_{\xi_{j} \xi_{j}}^{2} \Psi_{1,0, \phi_{j}} \\
+i\left[\partial_{t_{2}}^{2} \phi_{j}-\partial_{x_{2}}^{2} \phi_{j}\right] \Psi_{1,0, \phi_{j}}+\gamma\left|\Psi_{1,0, \phi_{j}}\right|^{2} \Psi_{1,0, \phi_{j}}=0 .
\end{array}
$$

It is convenient to write this equation in the form of ordinary differential equation in terms of characteristic variables

$$
\begin{array}{r}
\frac{d \Psi_{2,0, \phi_{j}}}{d \zeta_{j}}=-2 i \partial_{t_{2}} \phi_{j} \partial_{t_{2}} \Psi_{1,0, \phi_{j}}-\left[\left(\partial_{t_{1}} \xi_{j}\right)^{2}-\left(\partial_{x_{1}} \xi_{j}\right)^{2}\right] \partial_{\xi_{j}}^{2} \Psi_{1,0, \phi_{j}} \\
-i\left[\partial_{t_{2}}^{2} \phi_{j}-\partial_{x_{2}}^{2} \phi_{j}\right] \Psi_{1,0, \phi_{j}}-\gamma\left|\Psi_{1,0, \phi_{j}}\right|^{2} \Psi_{1,0, \phi_{j}} .
\end{array}
$$

Equation (3.6) shows that the right hand side of equation (3.7) does not depend on $\zeta_{j}$. To construct the bounded solution of (3.7) we demand the right hand side of the equation is equal to zero. It allows to determine the dependence of the leadingorder term on slow variable $t_{2}$. The amplitudes of the leading-order terms satisfy $(N-k)$ nonlinear Schrödinger equations

$$
\begin{array}{r}
2 i \partial_{t_{2}} \phi_{j} \partial_{t_{2}} \Psi_{1,0, \phi_{j}}+\left[\left(\partial_{t_{1}} \xi_{j}\right)^{2}-\left(\partial_{x_{1}} \xi_{j}\right)^{2}\right] \partial_{\xi_{j} \xi_{j}}^{2} \Psi_{1,0, \phi_{j}} \\
(3.8)+i\left[\partial_{t_{2}}^{2} \phi_{j}-\partial_{x_{2}}^{2} \phi_{j}\right] \Psi_{1,0, \phi_{j}}+\gamma\left|\Psi_{1,0, \phi_{j}}\right|^{2} \Psi_{1,0, \phi_{j}}=0, \quad j=k+1, \ldots, N
\end{array}
$$

The initial conditions for $\Psi_{1,0, \phi_{j}}$ are

$$
\begin{array}{r}
\left.\Psi_{1,0, \phi_{j}}\right|_{l_{k}=0}=\left.\Psi_{1,0, \phi_{j}}\right|_{l_{k}=+0}, j=k+2, \ldots, N \\
\left.\Psi_{1,0, \phi_{k+1} \mid}\right|_{l_{k+1}=0}=\int_{-\infty}^{\infty} d \sigma f_{k+1}\left(x_{1}\right) \exp \left(i \int_{0}^{\sigma} d \mu \lambda_{k+1}\left(x_{1}, t_{1}, \varepsilon\right)\right) .
\end{array}
$$

Integration in this integral is realized in the line of characteristic direction connected with (3.5).

The equations for the higher-order terms are obtained by the same manner

$$
\begin{gathered}
2 i\left(\partial_{t_{2}} \phi_{j} \partial_{t_{1}} \Psi_{n+1, k, \phi_{j}}-\partial_{x_{2}} \phi_{j} \partial_{x_{1}} \Psi_{n+1, k, \phi_{j}}\right)=2 i \partial_{t_{2}} \phi_{j} \partial_{t_{2}} \Psi_{n, k, \phi_{j}}-\partial_{\xi_{j} \xi_{j}}^{2} \Psi_{n, k, \phi_{j}} \\
-i\left[\partial_{t_{2}}^{2} \phi_{j}-\partial_{x_{2}}^{2} \phi_{j}\right] \Psi_{n, k, \phi_{j}}+\partial_{t_{1}} \xi_{j} \partial_{\xi t_{2}}^{2} \Psi_{n-1, k, \phi_{j}} \\
-\gamma \sum_{k_{1}, k_{2}, l_{1}, l_{2}, m_{1}, m_{2}, \alpha, \beta, \delta} \Psi_{k_{1}, k_{2}, \alpha} \Psi_{l_{1}, l_{2}, \beta} \Psi_{m_{1}, m_{2}, \delta},
\end{gathered}
$$

where $k_{1}+l_{1}+m_{1}=n+2, k_{2}+l_{2}+m_{2}=k, \alpha+\beta+\delta=\phi_{j}, \alpha \in \Upsilon_{k_{1}, k_{2}}^{k+1}, \beta \in$ $\Upsilon_{l_{1}, l_{2}}^{k+1}, \delta \in \Upsilon_{m_{1}, m_{2}}^{k+1}$. 
To construct the uniform asymptotic expansion with respect to $\zeta_{j}$ we obtain the linearized Schrodinger equation for higher-order term

$$
\begin{array}{r}
2 i \partial_{t_{2}} \phi_{j} \partial_{t_{2}} \Psi_{n, k, \phi_{j}}+\partial_{\xi_{j} \xi_{j}}^{2} \Psi_{n, k, \phi_{j}}+i\left[\partial_{t_{2}}^{2} \phi_{j}-\partial_{x_{2}}^{2} \phi_{j}\right] \Psi_{n, k, \phi_{j}} \\
\left(3.9=-\partial_{t_{1}} \xi \partial_{\xi_{j} t_{2}}^{2} \Psi_{n-1, k, \phi_{j}}-\gamma \sum_{k_{1}, k_{2}, l_{1}, l_{2}, m_{1}, m_{2}, \alpha, \beta, \delta} \Psi_{k_{1}, k_{2}, \alpha} \Psi_{l_{1}, l_{2}, \beta} \Psi_{m_{1}, m_{2}, \delta},\right.
\end{array}
$$

where $k_{1}+l_{1}+m_{1}=n+2, k_{2}+l_{2}+m_{2}=k, \alpha+\beta+\delta=\phi_{j}, \alpha \in \Upsilon_{k_{1}, k_{2}}^{k+1}, \beta \in$ $\Upsilon_{l_{1}, l_{2}}^{k+1}, \delta \in \Upsilon_{m_{1}, m_{2}}^{k+1}$

The amplitudes $\Psi_{n, k, \chi}$ as $\chi \in \Upsilon_{n, k}^{k+1^{\prime}}$ are determined by algebraic equations

$$
\left[-\left(\chi_{t_{2}}\right)^{2}+\left(\chi_{x_{2}}\right)^{2}+1\right] \Psi_{n, k, \chi}=F_{n, k, \chi}, \quad \chi \neq \pm \phi_{j}, j=1, \ldots, k .
$$

Here the right hand side of the equation depends on previous terms and their derivatives

$$
\begin{gathered}
F_{n, k, \chi}=-2 i \chi_{t_{2}} \partial_{t_{1}} \Psi_{n-1, k, \chi}+2 i \chi_{x_{2}} \partial_{x_{1}} \Psi_{n-1, k, \chi}-2 i \chi_{t_{2}} \partial_{t_{2}} \Psi_{n-2, k, \chi} \\
-i\left[\chi_{t_{2} t_{2}}-\chi_{x_{2} x_{2}}\right] \Psi_{n-2, k, \chi}-\partial_{t_{1} t_{2}}^{2} \Psi_{n-3, k, \chi}-\partial_{t_{2} t_{2}}^{2} \Psi_{n-4, k, \chi} \\
-\gamma \sum_{k_{1}, k_{2}, l_{1}, l_{2}, m_{1}, m_{2}, \alpha, \beta, \delta} \Psi_{k_{1}, k_{2}, \alpha} \Psi_{l_{1}, l_{2}, \beta} \Psi_{m_{1}, m_{2}, \delta},
\end{gathered}
$$

where $k_{1}+l_{1}+m_{1}=n-4, k_{2}+l_{2}+m_{2}=k, \alpha+\beta+\delta=\chi, \alpha \in \Upsilon_{k_{1}, k_{2}}^{k+1}, \beta \in$ $\Upsilon_{l_{1}, l_{2}}^{k+1}, \delta \in \Upsilon_{m_{1}, m_{2}}^{k+1}$.

Note. It's necessary to note that the multiplier $\left[-\left(\chi_{t_{2}}\right)^{2}+\left(\chi_{x_{2}}\right)^{2}+1\right]$ can be vanished on some curves. It leads to the resonances for the higher order terms of the asymptotics. The passage through the resonances does not change the leading-order terms of the asymptotic solution. We discuss this passage in section 6.

In this section we pay a special attention on the amplitudes $\Psi_{n, k, \chi}$ with the phase function $k S$. These amplitudes have the strongest order singularity on the curve $l_{k}=0$. The multiplier $\left[-\left(\chi_{t_{2}}\right)^{2}+\left(\chi_{x_{2}}\right)^{2}+1\right]$ on the left hand of equation (3.10) equals zero on the curve $l_{k}=0$. Here we explicitly write out a few first correction terms:

$$
\begin{gathered}
\Psi_{2,0, k S}=-\frac{f_{k}}{l_{k}} \\
\Psi_{3,0, k S}=2 i k \frac{\partial_{x_{1}} f_{k} \partial_{x_{2}} S}{l_{k}^{2}} \\
\Psi_{4,0, k S}=\frac{2 i k f_{k}\left[\partial_{t_{2}} S \partial_{t_{2}} l_{k}-\partial_{x_{2}} S \partial_{x_{2}} l_{k}\right]-4 k^{2}\left(\partial_{x_{2}} S\right)^{2} \partial_{x_{1}}^{2} f_{k}}{l_{k}^{3}}- \\
\frac{2 i k \partial_{t_{2}} f_{k} \partial_{t_{2}} S+\partial_{x_{1}}^{2} f_{k}+i k \partial_{t_{2}}^{2} S f_{k}}{l_{k}^{2}} .
\end{gathered}
$$

The formula for the $n$-th order term has the form

$$
\begin{gathered}
\Psi_{n, m, k S}=\frac{1}{l_{k}}\left[\partial_{t_{2}}^{2} \Psi_{n-4, m, k S}+2 i k \partial_{t_{2}} S \partial_{t_{2}} \Psi_{n-2, m, k S}+i k S_{t_{2} t_{2}} \Psi_{n-2, m, k S}-\right. \\
2 i k \partial_{x_{2}} S \partial_{x_{2}} \Psi_{n-2, m, k S}-i k \partial_{x_{2}}^{2} S \Psi_{n-2, m, k S}-\partial_{x_{1}}^{2} \Psi_{n-2, m, k S}- \\
2 \partial_{x_{1} x_{2}}^{2} \Psi_{n-3, m, k S}-\partial_{x_{2}}^{2} \Psi_{n-4, m, k S}-2 i k \partial_{x_{2}} S \partial_{x_{1}} \Psi_{n-1, m, k S}+
\end{gathered}
$$




$$
\begin{aligned}
& \left.\gamma \sum_{\substack{n_{1}+n_{2}+n_{3}=n, k_{1}+k_{2}+k_{3}=k S \\
m_{1}+m_{2}+m_{3}=m}} \Psi_{n_{1}, m_{1}, k_{1}} \Psi_{n_{2}, m_{2}, k_{2}} \Psi_{n_{3}, m_{3}, k_{3}}\right] . \\
& m_{1}
\end{aligned}
$$

The main order of the singularity corresponds to the terms when $m=0$. This order depends on the number of correction term.

LEMma 3.1. The coefficient $\Psi_{n, m, \varphi}, \varphi \in \Upsilon_{1,0}^{k+1} \cup\{k S\}$ has the following behaviour

$$
\Psi_{n, m, \varphi}=O\left(l_{k}^{-(n-2 m-1)}\right), \quad l_{k} \rightarrow-0,
$$

Proof. At first we prove formula (3.16) for the phase $\varphi=k S$. The validity of formula 3.16 for $n=2,3,4$ and $m=0$ directly obtains from 3.12), 3.13), (3.14). Suppose now that this formula is valid for the term $\Psi_{n-1,0, \varphi}$. The increase of the order of the singularity as $l_{k} \rightarrow 0$ takes place due to differentiation with respect to $x_{2}, t_{2}$ and the nonlinear term in formula (3.15). Differentiation of the terms in formula (3.15) leads to formula (3.16) for $m=0$.

The validity for values $m \geq 1$ and other values of $\varphi$ can be obtained by direct calculations as it was shown above. Lemma is proved.

These lemma allows us to write the asymptotic representation for the coefficients

$$
\Psi_{n, m, \varphi}=\sum_{j=-(n-2 m-1)}^{\infty} \Psi_{n, m, \varphi}^{j} l_{k}^{j}, \quad \varphi \in \Upsilon_{1,0}^{k+1} \cup\{k S\}, l_{k} \rightarrow-0 .
$$

The terms $\Psi_{n, m, \varphi}$ for $\varphi \notin \Upsilon_{1,0}^{k+1} \cup\{k S\}$ have the more weaker order of the singularity as $l_{k} \rightarrow-0$. Equations (3.15) for these values of $\varphi$ have the same structure but the multiplier $\frac{1}{l_{j}}$ is regular as $l_{k} \rightarrow-0$.

The domain of the validity as $l_{k} \rightarrow-0$ for the formal asymptotic solution in the form 3.1 follows from the relation

$$
\frac{\varepsilon \Psi_{n+1}^{k+1}}{\Psi_{n}^{k+1}} \ll 1 .
$$

It yields

$$
-l_{k} \gg \varepsilon
$$

The following theorem is proved.

TheOREM 3.2. In the domain $-l_{k} \gg \varepsilon, l_{k+1} \gg \varepsilon$ the formal asymptotic solution of equation (2.1) has form (3.1). The coefficients of the asymptotics are defined either from differential equations (3.8), (3.9) or from algebraic equations (3.12), 3.13, 3.14, 3.15.

\section{Internal asymptotics}

This part of the paper contains the asymptotic construction of the solution for equation 2.1] in the neighborhood of the curve $l_{k}=0$. The domain of validity of this internal asymptotics intersects with domain of validity of expansion (3.1). 
These expansions are matched. Here we present asymptotic constructions for arbitrary $k$ in details. It is necessary to note this section also describes the transition from the forced oscillations of $O\left(\varepsilon^{2}\right)$ to the solution of $O(\varepsilon)$ when $k=N$.

Let us construct the internal asymptotic expansion in the domain $\left|l_{k}\right| \ll 1$. Denote

$$
\lambda_{k}\left(x_{1}, t_{1}, \varepsilon\right)=\frac{1}{\varepsilon} l_{k}\left(\varepsilon x_{1}, \varepsilon t_{1}\right) .
$$

THEOREM 4.1. In the domain $\left|l_{k}\right| \ll 1$ the formal asymptotic solution for equation (2.1) has the form

$$
U(x, t, \varepsilon)=\varepsilon W_{1}+\sum_{n=2}^{\infty} \varepsilon^{n} W_{n},
$$

where

$$
\begin{gathered}
W_{1}=W_{1,0, k S} \exp \left\{i k S\left(t_{2}, x_{2}\right) / \varepsilon^{2}\right\}+\sum_{m=k+1}^{N} \sum_{ \pm \phi_{m}} \exp \left\{ \pm i \phi_{m}\left(x_{2}, t_{2}\right) / \varepsilon^{2}\right\} W_{1,0, \pm \phi_{m}} \\
W_{n}=\sum_{j=0}^{n-2} \ln ^{j}(\varepsilon) W_{n, j, k S} \exp \left(i k \frac{S\left(t_{2}, x_{2}\right)}{\varepsilon^{2}}\right) \\
+\sum_{j=0}^{n-2} \ln ^{j}(\varepsilon)\left(\sum_{m=k+1}^{N} \sum_{ \pm \phi_{m}} \exp \left\{ \pm i \phi_{m}\left(x_{2}, t_{2}\right) / \varepsilon^{2}\right\} W_{n, j, \pm \phi_{m}}\left(x_{1}, t_{1}, t_{2}\right)\right. \\
\left.+\sum_{\chi \in \Upsilon_{n, j}^{\prime}} \exp \left\{i \chi\left(x_{2}, t_{2}\right) / \varepsilon^{2}\right\} W_{n, j, \chi}\left(x_{1}, t_{1}, t_{2}\right)\right),
\end{gathered}
$$

where $\Upsilon_{n, j}^{\prime}$ is the set of phase functions which is determined by

$$
\begin{aligned}
& \Upsilon_{1,0}= \pm \phi_{k+1}, \pm \phi_{k+2}, \ldots, \pm \phi_{N}, \pm k S ; \\
& \Upsilon_{2,0}= \pm \phi_{k+1}, \pm \phi_{k+2}, \ldots, \pm \phi_{N}, \pm S, \ldots, \pm N S \\
& \Upsilon_{n, j}=\bigcup \quad \chi_{n_{1}, j_{1}}+\chi_{n_{2}, j_{2}}+\chi_{n_{3}, j_{3}}, \quad \chi_{n_{p}, j_{p}} \in \Upsilon_{n_{p}, j_{p}} . \\
& n_{1}+n_{2}+n_{3}=n \text {, } \\
& j_{1}+j_{2}+j_{3}=j \\
& \Upsilon_{n, j}^{\prime}=\Upsilon_{n, j} \backslash \Upsilon_{1,0} .
\end{aligned}
$$

The function $W_{n, j, k S}$ is solution of the problem for equation (4.8) with zero condition as $\lambda_{k} \rightarrow-\infty$.

There is a difference between asymptotics 4.2 and external pre-resonance asymptotics (3.1). Asymptotic expansion (4.2) defines the generation of the $k$-th mode by the local resonance for the leading-order term of the asymptotic expansion. The difference is the collection of phase function. The collection of phases $\Upsilon_{1,0}$ contains $(N-k+1)$ phases but the phase $\pm \phi_{k}$ does not exist in the $k$-th resonant layer. There phases $\pm k S$ are presented in the $\Upsilon_{1,0}$ and $\Upsilon_{2,0}$ collections.

The proof of theorem 4.1 consists in three steps. First we derive equations for the coefficients of the asymptotics. Second we solve the problems for the coefficients. And third we determine the domain of the validity for expansion 4.2). 
4.1. The equations for coefficients. In the domain $1 \ll \lambda_{k} \ll \varepsilon^{-1}$ both asymptotics (3.1) and 4.2 are valid. This fact allows us to obtain the asymptotic representation for coefficients of (4.2). Substitute $l_{k}=\varepsilon \lambda_{k}$ in formula (3.17) and expand the obtained expression with respect to powers of $\varepsilon$. It yields

$$
W_{n, m, \varphi}=\sum_{j=n-2 m-1}^{\infty} \lambda_{k}^{-j} \Psi_{n+1, m, \varphi}^{j}\left(x_{2}, t_{2}, x_{1}\right), \quad \lambda_{k} \rightarrow-\infty .
$$

Let us obtain the differential equations for the coefficients of asymptotics 4.2 . Substitute (4.2) in equation (2.1) and collect the terms with equal powers of small parameter and exponents. It yields the equations for coefficients $W_{n, j, \varphi}, \varphi \in \Upsilon_{n, j}$. In particularly, the terms of the order $\varepsilon^{2}$ give us the equations for the amplitudes of the leading-order terms

$$
2 i k \partial_{t_{2}} S \partial_{t_{1}} W_{1,0, k S}-2 i k \partial_{x_{2}} S \partial_{x_{1}} W_{1,0, k S}-\lambda_{k} W_{1,0, k S}=f_{k},
$$

$2 i \partial_{t_{2}} \phi_{j} \partial_{t_{1}} W_{1,0, \phi_{j}}-2 i \partial_{x_{2}} \phi_{j} \partial_{x_{1}} W_{1,0, \phi_{j}}-l_{\phi_{j}}\left(x_{2}, t_{2}\right) W_{1,0, \phi_{j}}=0, \quad j=k+1, \ldots, N$. and complex conjugated equation for $W_{1,0,-\varphi}, \varphi \in \Upsilon_{1,0}(k)$.

The relation of the order $\varepsilon^{3}$ in equation (2.1) gives $2(N-k)+2$ equations:

$$
\begin{gathered}
2 i k \partial_{t_{2}} S \partial_{t_{1}} W_{2,0, k S}-2 i k \partial_{x_{2}} S \partial_{x_{1}} W_{2,0, k S}-\lambda_{k} W_{2,0, k S}=\partial_{x_{1}}^{2} W_{1,0, k S}-\partial_{t_{1}}^{2} W_{1,0, k S} \\
-i k\left[\partial_{t_{2}}^{2} S-\partial_{x_{2}}^{2} S\right] W_{1,0, k S}-2 i k \partial_{t_{2}} S \partial_{t_{2}} W_{1,0, k S}+2 i k \partial_{x_{2}} S \partial_{x_{2}} W_{1,0, k S} \\
-3 \gamma\left(\left|W_{1,0, k S}\right|^{2}+\sum_{m=k+1}^{N}\left|W_{1,0, \phi_{m}}\right|^{2}\right) W_{1,0, k S} . \\
=\partial_{x_{1}}^{2} W_{1,0, \phi_{j}}-\partial_{t_{1}}^{2} W_{1,0, \phi_{j}}--i\left[\partial_{t_{2}}^{2} \phi_{j}-\partial_{x_{2}}^{2} \phi_{j}\right] W_{1,0, \phi_{j}}-2 i \partial_{t_{2}} \phi_{j} \partial_{t_{2}} W_{1,0, \phi_{j}} \\
4.6){ }_{t_{t_{1}}} W_{2,0, \phi_{j}}-2 i \partial_{x_{2}} \phi_{j} \partial_{x_{1}} W_{2,0, \phi_{j}}-l_{\phi_{j}}\left(x_{2}, t_{2}\right) W_{2,0, \phi_{j}} \\
+2 i \partial_{x_{2}} S \partial_{x_{2}} W_{1,0, \phi_{j}}-3 \gamma\left(\left|W_{1,0, k S}\right|^{2}+\sum_{m=k+1}^{N}\left|W_{1,0, \phi_{m}}\right|^{2}\right) W_{1,0, \phi_{j}} .
\end{gathered}
$$

The higher-order terms are calculated by the same way. In particularly $W_{n, j, \varphi}, \varphi \in$ $\Upsilon_{1,0}$ is determined by differential equations. Here we represent the equation for amplitude under $\varphi=k S$. The equations for other phases $\varphi \in \Upsilon_{1,0}$ can be obtained in a similar.

$$
2 i k \partial_{t_{2}} S \partial_{t_{1}} W_{n, j, k S}-2 i k \partial_{x_{2}} S \partial_{x_{1}} W_{n, j, k S}-\lambda_{k} W_{n, j, k S}=F_{n, j, k} .
$$

The right hand side of equation 4.8 has the form

$$
\begin{gathered}
F_{n, j, k}=-2 i k \partial_{t_{2}} S \partial_{t_{2}} W_{n-1, j, k S}+2 i k \partial_{x_{2}} S \partial_{x_{2}} W_{n-1, j, k S}+k^{2}\left(\partial_{t_{2}} S\right)^{2} W_{n-1, j, k S} \\
-k^{2}\left(\partial_{x_{2}} S\right)^{2} W_{n-1, j, k S}-\partial_{t_{1}}^{2} W_{n-1, j, k S}+\partial_{x_{1}}^{2} W_{n-1, j, k S}-\partial_{t_{2}} \partial_{t_{1}} W_{n-2, j, k S} \\
+\partial_{x_{2}} \partial_{x_{1}} W_{n-2, j, k S}-\partial_{t_{2}}^{2} W_{n-3, j, k S}+\partial_{x_{2}}^{2} W_{n-3, j, k S}
\end{gathered}
$$




$$
\begin{gathered}
-\gamma \quad \sum_{n_{1}, j_{1}, \chi_{1}} W_{n_{2}, j_{2}, \chi_{2}} W_{n_{3}, j_{3}, \chi_{3}} . \\
n_{1}+n_{2}+n_{3}=n+1, \\
j_{1}+j_{2}+j_{3}=j, \\
\chi_{1}+\chi_{2}+\chi_{3}=k S, \\
\chi_{j} \in \Upsilon_{n_{j}}(k), j=1,2,3
\end{gathered}
$$

The amplitudes $W_{n, k, \chi}$ as $\chi \notin \Upsilon_{1,0}$ are determined by algebraic equations

$$
\left[-\left(\chi_{t_{2}}\right)^{2}+\left(\chi_{x_{2}}\right)^{2}+1\right] W_{n, k, \chi}=F_{n, k, \chi} .
$$

Here the right hand side of the equation depends on previous terms and their derivatives

$$
\begin{gathered}
F_{n, k, \chi}=-2 i \chi_{t_{2}} \partial_{t_{1}} W_{n-1, k, \chi}+2 i \chi_{x_{2}} \partial_{x_{1}} W_{n-1, k, \chi}-2 i \chi_{t_{2}} \partial_{t_{2}} W_{n-2, k, \chi} \\
-i\left[\chi_{t_{2} t_{2}}-\chi_{x_{2} x_{2}}\right] W_{n-2, k, \chi}-\partial_{t_{1} t_{2}}^{2} W_{n-3, k, \chi}-\partial_{t_{2} t_{2}}^{2} W_{n-4, k, \chi} \\
-\gamma \sum_{N, k_{2}, l_{1}, l_{2}, m_{1}, m_{2}, \alpha, \beta, \delta} W_{N, k_{2}, \alpha} W_{l_{1}, l_{2}, \beta} W_{m_{1}, m_{2}, \delta},
\end{gathered}
$$

where $N+l_{1}+m_{1}=n-4, k_{2}+l_{2}+m_{2}=k, \alpha+\beta+\delta=\chi, \alpha \in \Upsilon_{N, k_{2}}, \beta \in$ $\Upsilon_{l_{1}, l_{2}}, \delta \in \Upsilon_{m_{1}, m_{2}}$.

4.2. The solvability of equations for higher-order terms. In this section we present the explicit form for the higher-order term $W_{n, j, k S}$ and investigate the asymptotic behaviour as $\lambda_{k} \rightarrow \pm \infty$.

4.2.1. Characteristic variables. The function $W_{n, j, k S}$ satisfies equation (4.8). The solution is constructed by characteristic method. Define the characteristic variables $\sigma, \xi$. We choose a point $\left(x_{1}^{0}, t_{1}^{0}\right)$ such that $\left.\partial_{x_{2}} l_{k}\right|_{\left(x_{1}^{0}, t_{1}^{0}\right)} \neq 0$ as origin and denote by $\sigma$ the variable along the characteristic family for equation 4.8). We suppose $\sigma=0$ on the curve $\lambda_{k}=0$. The variable $\xi$ mensurates the distance along the curve $\lambda_{k}=0$ from the point $\left(x_{1}^{0}, t_{1}^{0}\right)$. This point $\left(x_{1}^{0}, t_{1}^{0}\right)$ corresponds to $\xi=0$. Let the positive direction for parameter $\xi$ coincides with the positive direction of $x_{2}$ in the neighborhood of $\left(x_{1}^{0}, t_{1}^{0}\right)$.

The characteristic equations for 4.8 have a form

$$
\frac{d t_{1}}{d \sigma}=2 k \partial_{t_{2}} S\left(\varepsilon x_{1}, \varepsilon t_{1}\right), \quad \frac{d x_{1}}{d \sigma}=-2 k \partial_{x_{2}} S\left(\varepsilon x_{1}, \varepsilon t_{1}\right) .
$$

The initial conditions for the equations are

$$
\left.x_{1}\right|_{\sigma=0}=x_{1}^{0},\left.\quad t_{1}\right|_{\sigma=0}=t_{1}^{0} .
$$

Lemma 4.2. The Cauchy problem (4.12), (4.13) for characteristics has a solutions as $|\sigma|<c_{1} \varepsilon^{-1}, \quad c_{1}=$ const $>0$.

Proof. The Cauchy problem 4.12, 4.13 is equivalent to the system of the integral equations

$$
t_{1}=t_{1}^{0}+2 \int_{0}^{\sigma} k \partial_{t_{2}} S\left(\varepsilon x_{1}, \varepsilon t_{1}\right) d \zeta, \quad x_{1}=x_{1}^{0}-2 \int_{0}^{\sigma} k \partial_{x_{2}} S\left(\varepsilon x_{1}, \varepsilon t_{1}\right) d \zeta .
$$

Substitute $\tilde{t}_{2}=\left(t_{1}-t_{1}^{0}\right) \varepsilon, \tilde{x}_{2}=\left(x_{1}-x_{1}^{0}\right) \varepsilon$. It yields

$\tilde{t}_{2}=2 \int_{0}^{\varepsilon \sigma} k \partial_{t_{2}} S\left(\tilde{x}_{2}-\varepsilon x_{1}^{0}, \tilde{t}_{2}-\varepsilon t_{1}^{0}\right) d \zeta, \quad \tilde{x}_{2}=-2 \int_{0}^{\varepsilon \sigma} k \partial_{x_{2}} S\left(\tilde{x}_{2}-\varepsilon x_{1}^{0}, \tilde{t}_{2}-\varepsilon t_{1}^{0}\right) d \zeta$. 
The integrands are smooth and bounded functions on the plane $x_{2}, t_{2}$. There exists the constant $c_{1}=$ const $>0$ such that the integral operator is the contraction operator as $\varepsilon|\sigma|<c_{1}$. Lemma 4.2 is proved.

Assumption. We assume that the change of variables $\left(x_{1}, t_{1}\right) \rightarrow(\sigma, \xi)$ is unique in the neighborhood of the curve $\lambda_{k}=0$. This assumption means that the characteristics for equation (4.8) do not touch the curve $\lambda_{k}=0$. It means

$$
\partial_{x_{2}} l_{k} \partial_{x_{2}} S-\partial_{t_{2}} l_{k} \partial_{t_{2}} S \neq 0 .
$$

It is convenient to use the following asymptotic formulas for change of variables $\left(x_{1}, t_{1}\right) \rightarrow(\sigma, \xi)$.

LEMMA 4.3. In the domain $|\sigma| \ll \varepsilon^{-1}$ the asymptotics as $\varepsilon \rightarrow 0$ of the solutions for Cauchy problem (4.12), (4.13) have the form

$$
\begin{array}{r}
x_{1}\left((\text { \&.. } \$ 5 \xi)-x_{1}^{0}(\xi)=-2 \sigma k \partial_{x_{2}} S+2 \sum_{n=1}^{N} \varepsilon^{n} \sigma^{n+1} g_{n}\left(\varepsilon x_{1}, \varepsilon t_{1}\right)+O\left(\varepsilon^{N+1} \sigma^{N+2}\right),\right. \\
\left(t_{4} .(16) \xi, \varepsilon\right)-t_{1}^{0}(\xi)=2 \sigma k \partial_{t_{2}} S+2 \sum_{n=1}^{N} \varepsilon^{n} \sigma^{n+1} h_{n}\left(\varepsilon x_{1}, \varepsilon t_{1}\right)+O\left(\varepsilon^{N+1} \sigma^{N+2}\right),
\end{array}
$$

where

$$
g_{n}=-\left.k \frac{d^{n}}{d \sigma^{n}}\left(\partial_{x_{2}} S\right)\right|_{\sigma=0}, \quad h_{n}=\left.k \frac{d^{n}}{d \sigma^{n}}\left(\partial_{t_{2}} S\right)\right|_{\sigma=0} .
$$

The lemma proves by integration by parts of equations 4.14.

The next proposition gives us the asymptotic formula which connects variables $\sigma$ and $\lambda_{k}$ as $\sigma, \lambda_{k} \rightarrow \pm \infty$.

Lemma 4.4. Let be $\sigma \ll \varepsilon^{-1}$, then:

$$
\lambda_{k}=\phi(\xi) \sigma+O\left(\varepsilon \sigma^{2}\right), \quad \phi(\xi)=\left.\frac{d \lambda_{k}}{d \sigma}\right|_{\sigma=0} \quad \sigma \rightarrow \infty
$$

Proof. From formula 4.1 we obtain the representation in the form

$$
\lambda_{k}=\sum_{j=1}^{\infty} \lambda_{k}^{j}\left(x_{1}, t_{1}, \varepsilon\right) \sigma^{j} \varepsilon^{j-1},
$$

where

$$
\lambda_{k}^{j}\left(x_{1}, t_{1}, \varepsilon\right)=\left.\frac{1}{j !} \frac{d^{j}}{d \sigma^{j}} \lambda_{k}\left(x_{1}, t_{1}, \varepsilon\right)\right|_{\sigma=0} .
$$

It yields

$$
\lambda_{k}=\left.\frac{d \lambda_{k}}{d \sigma}\right|_{\sigma=0} \sigma+O\left(\varepsilon \sigma^{2} \frac{d^{2} \lambda_{k}}{d \sigma^{2}}\right) .
$$

Let be

$$
\left|\frac{d^{2} l_{k}}{d \sigma^{2}}\right| \geq \text { const, } \xi \in R .
$$

The function $d \lambda_{k} / d \sigma$ is not equal to zero

$$
\frac{d \lambda_{k}}{d \sigma}=\frac{1}{2}\left(-k \partial_{x_{2}} \lambda_{k} \partial_{x_{2}} S+k \partial_{t_{2}} \lambda_{k} \partial_{t_{2}} S\right) \neq 0 .
$$


Let us suppose $d \lambda_{k} / d \sigma>0$. It yields

$$
\lambda_{k}=\phi(\xi) \sigma+O\left(\varepsilon \sigma^{2}\right), \quad \phi(\xi)=\left.\frac{d \lambda_{k}}{d \sigma}\right|_{\sigma=0}
$$

The lemma is proved.

4.2.2. Solutions of the equations for higher-order terms. The higher-order terms $W_{n, j, \pm k S}$ are solutions of equation 4.8 with the given asymptotic behaviour $\lambda_{k} \rightarrow$ $-\infty$. Equation 4.8 can be written in terms of characteristic variables as

$$
i \frac{d}{d \sigma} W_{n, j, k S}-\lambda_{k} W_{n, j, k S}=F_{n, j, k S} .
$$

Lemma 4.5. The solution of equation (4.8) with the asymptotic behaviour (4.3) as $\lambda_{k} \rightarrow-\infty$ has a form

$$
\begin{array}{r}
W_{n, j, k S}=\exp \left(-i \int_{0}^{\sigma} d \zeta \lambda_{k}\left(x_{1}, t_{1}, \varepsilon\right)\right) \\
\times \int_{-\infty}^{\sigma} d \zeta F_{n, j, k S}\left(x_{1}, t_{1}, \varepsilon\right) \exp \left(-i \int_{0}^{\zeta} d \chi \lambda_{k}\left(x_{1}, t_{1}, \varepsilon\right)\right) .
\end{array}
$$

Proof. By direct substitution we see that expression (4.18) is the solution of (4.17). The asymptotics of this solution as $\lambda_{k} \rightarrow-\infty$ can be obtained by integration by parts and substitution

$$
\frac{d}{d \sigma}=2 k \partial_{t_{2}} S \partial_{t_{1}}-2 k \partial_{x_{2}} S \partial_{x_{1}}
$$

It yields

$$
W_{n, j, k S}=\sum_{j=0}^{\infty}\left(\frac{2 k \partial_{t_{2}} S \partial_{t_{1}}-2 k \partial_{x_{2}} S \partial_{x_{1}}}{i \lambda_{k}}\right)^{j}\left[\frac{F_{n, j, k S}}{i \lambda_{k}}\right], \quad \lambda_{k} \rightarrow-\infty .
$$

From formula 4.9 we obtain that formulas 4.19 and 4.3 are equivalent. The lemma is proved.

4.3. Asymptotics as $\lambda_{k} \rightarrow \infty$ and domain of validity of the internal asymptotics. The domain of validity of the internal expansion is determined by the asymptotics of higher-order terms. In this section we show that the $n$-th order term of the asymptotic solution increases as $\lambda_{k}^{n-1}$ when $\lambda_{k} \rightarrow \infty$. This increase of higher-order terms allows us to determine the domain of validity for internal asymptotics 4.2 as $\lambda_{k} \rightarrow \infty$.

4.3.1. Asymptotics of higher-order terms. This section contains two propositions concerning asymptotic behaviour as $\lambda_{k} \rightarrow \infty$ for higher-order terms in (4.2). The first lemma describes the asymptotic behaviour of higher-order terms as $\lambda_{k} \rightarrow \infty$ and the second one contains a result about asymptotics of the phase function.

Lemma 4.6. The asymptotic behaviour of $W_{n, j, k S}$ as $1 \ll \lambda_{k} \ll \varepsilon^{-1}$ has a form

$$
\begin{array}{r}
W_{n, j, k S}=\sum_{j=0}^{n-1} \sum_{m=0}^{j-1}\left(\lambda_{k}^{j} \ln ^{m}\left|\lambda_{k}\right| W_{n, j, k S}^{(j, m)}(\xi)\right) \exp \left(-i \int_{0}^{\sigma} d \zeta \lambda_{k}\left(x_{1}, t_{1}, \varepsilon\right)\right) \\
+\sum_{m=0}^{\infty}\left(\frac{2 k \partial_{t_{2}} S \partial_{t_{1}}-2 k \partial_{x_{2}} S \partial_{x_{1}}}{i \lambda_{k}}\right)^{m}\left[\frac{F_{m, j, k S}}{i \lambda_{k}}\right] .
\end{array}
$$


Proof. Let us calculate the asymptotics of the leading-order term

$$
\begin{gathered}
W_{1,0, k S}=\exp \left(-i \int_{0}^{\sigma} d \zeta \lambda_{k}\left(x_{1}, t_{1}, \varepsilon\right)\right) \int_{-\infty}^{\zeta} d \zeta f_{k}\left(x_{1}\right) \exp \left(i \int_{0}^{\sigma} d \chi \lambda_{k}\left(x_{1}, t_{1}, \varepsilon\right)\right) \\
=\exp \left(-i \int_{0}^{\sigma} d \zeta \lambda_{k}\left(x_{1}, t_{1}, \varepsilon\right)\right) \int_{-\infty}^{\infty} d \zeta f_{k}\left(x_{1}\right) \exp \left(i \int_{0}^{\zeta} d \chi \lambda_{k}\left(x_{1}, t_{1}, \varepsilon\right)\right) \\
-\exp \left(-i \int_{0}^{\sigma} d \zeta \lambda_{k}\left(x_{1}, t_{1}, \varepsilon\right)\right) \int_{-\sigma}^{\infty} d \zeta f_{k}\left(x_{1}\right) \exp \left(i \int_{0}^{\zeta} d \chi \lambda_{k}\left(x_{1}, t_{1}, \varepsilon\right)\right) .
\end{gathered}
$$

Further by integration by parts of the last term we obtain formula 4.20 as $n=1$, where

$$
\begin{gathered}
W_{1,0, k S}^{(0,0)}(\xi)=\int_{-\infty}^{\infty} d \sigma f_{k}\left(x_{1}\right) \exp \left(i \int_{0}^{\sigma} d \chi \lambda_{k}\left(x_{1}, t_{1}, \varepsilon\right)\right) \\
F_{1,0, k S}=f_{k}\left(x_{1}\right) .
\end{gathered}
$$

To calculate the asymptotics of $W_{2,0, k S}$ in formula 4.18 we use the asymptotics with respect to $\sigma$ of the leading-order term. Integral 4.18 contains the term with linear increase with respect to $\sigma$ when $n=2$. We eliminate this growing part from integral explicitly. The residuary integral converges as $\sigma \rightarrow \infty$. It can be calculated in the same manner as it was calculated for $W_{1,0, k S}$. It yields formula (4.20 as $n=2$, where

$$
W_{2,0, k S}^{(1,0)}(\xi)=W_{1,0, k S}^{(0,0)}(\xi) \text {. }
$$

The same direct calculations are realized for the $n$-th order term. The lemma is proved.

To complete the proof of theorem 4.1 we need to obtain the domain of validity of asymptotics 4.2. The formal series (4.2) is asymptotic when

$$
\frac{\varepsilon W_{n+1}}{W_{n}} \ll 1, \quad \varepsilon \rightarrow 0 .
$$

Lemma 4.6 gives $\lambda_{k} \ll \varepsilon^{-1}$. After substitution $\lambda_{k}=\varepsilon l_{k}$ we obtain $l_{k} \ll 1$. Theorem 4.1 is proved.

4.3.2. Asymptotics of the phase function as $\lambda_{k} \rightarrow \infty$. To obtain the asymptotics as $\lambda_{k} \rightarrow \infty$ we need to derive the asymptotics of the phase function in formula 4.20 .

LEMMA 4.7. As $\lambda_{k} \rightarrow \infty$ :

$$
\int_{0}^{\sigma} d \xi \lambda_{k}=\frac{S}{\varepsilon^{2}}+\frac{1}{\varepsilon}\left(\partial_{x_{2}} S\left(x_{1}-x_{1}^{0}\right)+\partial_{t_{2}} S\left(t_{1}-t_{1}^{0}\right)\right)+O\left(\varepsilon \lambda_{k}^{3}\right) .
$$

Proof. Substitute the asymptotics of $\lambda_{k}$ from lemma 4.6. Calculate the asymptotics of the integral in formula 4.21

$$
\begin{gathered}
\int_{0}^{\sigma} d \zeta \lambda_{k}\left(x_{1}, t_{1}, \varepsilon\right)=\int_{0}^{\sigma} \frac{d \zeta}{2}\left[\left(-k \partial_{x_{2}} l_{k} \partial_{x_{2}} S+k \partial_{t_{2}} l_{k} \partial_{t_{2}} S\right) \zeta+O\left(\varepsilon \zeta^{2}\right)\right] \\
=\left(-k \partial_{x_{2}} l_{k} \partial_{x_{2}} S+k \partial_{t_{2}} l_{k} \partial_{t_{2}} S\right) \frac{\sigma^{2}}{4}+O\left(\varepsilon \sigma^{3}\right) .
\end{gathered}
$$

The asymptotics of the phase function $k S\left(x_{2}, t_{2}\right)$ in the neighborhood of the curve $l_{1}=0$ is represented by a segment of the Taylor series. It yields

$$
\frac{k S}{\varepsilon^{2}}=\frac{1}{\varepsilon}\left(k \partial_{x_{2}} S\left(x_{1}-x_{1}^{0}\right)+k \partial_{t_{2}} S\left(t_{1}-t_{1}^{0}\right)\right)
$$




$$
\begin{aligned}
+\frac{1}{2}\left(k S_{x_{2} x_{2}}\left(x_{1}-x_{1}^{0}\right)^{2}\right. & \left.+2 k S_{x_{2} t_{2}}\left(x_{1}-x_{1}^{0}\right)\left(t_{1}-t_{1}^{0}\right)+k S_{t_{2} t_{2}}\left(t_{1}-t_{1}^{0}\right)^{2}\right) \\
+ & O\left(\varepsilon\left(\left|t_{1}-t_{1}^{0}\right|+\left|t_{1}-t_{1}^{0}\right|\right)^{3}\right) .
\end{aligned}
$$

Substitute instead of $\left(x_{1}-x_{1}^{0}\right)$ and $\left(t_{1}-t_{1}^{0}\right)$ their asymptotic behaviour with respect to $\varepsilon$ from lemma 4.3 . This substitution and result of lemma 4.4 complete the proof of lemma 4.7 .

The asymptotics as $\lambda_{k} \rightarrow-\infty$ contains fast oscillating terms with phase functions $\phi_{k+1}, \ldots, \phi_{N}, m S, m \in Z, m \neq k$. The leading-order term of the asymptotics as $\lambda_{k} \rightarrow \infty$ contains the oscillations with an additional phase function. We obtain this result from lemma 4.6. Denote this new phase function by $\phi_{k}\left(x_{2}, t_{2}\right) / \varepsilon^{2}$. The asymptotics of this function is obtained in lemma 4.7. The nonlinearity and additional phase function lead to more complicated structure of the phase set for higher-order terms of the asymptotics as $\lambda_{k} \rightarrow \infty$.

LEMMA 4.8. The phase set $\Upsilon_{n, j}$ for the $n$-th order term of the asymptotics as $\lambda_{k} \rightarrow \infty$ is determined by formula

$$
\begin{gathered}
\Upsilon_{1,0}=\left\{ \pm \phi_{k}, \ldots, \pm \phi_{N}\right\} ; \quad \Upsilon_{2,0}=\left\{ \pm \phi_{k}, \ldots, \pm \phi_{N}, \pm S, \ldots, \pm N S\right\}, \\
\Upsilon_{n, j}=\cup \chi_{n_{1}, j_{1}}+\chi_{n_{2}, j_{2}}+\chi_{n_{3}, j_{3}}, \quad \chi_{n_{m}, j_{m}} \in \Upsilon_{n_{m}, j_{m}}, \\
\text { where } n_{1}+n_{2}+n_{3}=n, j_{1}+j_{2}+j_{3}=j .
\end{gathered}
$$

The proof of this lemma follows from the asymptotic formula for $n$-th order term. Representation (4.2), formula (4.20) and lemma 4.6 allow us to construct the asymptotics as $\lambda \rightarrow \infty$ of the internal expansion in an explicit form

$$
\begin{gathered}
U=\sum_{n=1}^{N} \varepsilon^{n} \sum_{p=0}^{n-2} \ln ^{p}(\varepsilon)\left(\sum_{j=0}^{n-1} \sum_{m=0}^{n-2} \lambda^{j} \ln ^{m}|\lambda| W_{n, p, k}^{(j, m)}(\xi)\right) \\
\times \exp \left[-i\left(\frac{k}{\varepsilon}\left(\partial_{x_{2}} S\left(x_{1}-x_{1}^{0}\right)+\partial_{t_{2}} S\left(t_{1}-t_{1}^{0}\right)\right)+O\left(\varepsilon \lambda^{3}\right)\right)\right] \\
+\sum_{n=1}^{N} \varepsilon^{n} \sum_{p=0}^{n-2} \ln ^{p}(\varepsilon)\left(\sum_{j=0}^{\infty}\left(\frac{2 k \partial_{t_{2}} S \partial_{t_{1}}-2 k \partial_{x_{2}} S \partial_{x_{1}}}{i \lambda}\right)^{j}\left[\frac{F_{n, p, k S}}{i \lambda}\right]\right) \exp \left\{i k \frac{S\left(t_{2}, x_{2}\right)}{\varepsilon^{2}}\right\} \\
(4.22)+\sum_{n=2}^{N} \varepsilon^{n} \sum_{p=0}^{n-2} \ln ^{p}(\varepsilon)\left(\sum_{\varphi \in \Upsilon_{n, p} \backslash k S} W_{n, p, \varphi} \exp \left\{i \frac{\varphi\left(t_{2}, x_{2}\right)}{\varepsilon^{2}}\right\}\right)+\text { c.c. }
\end{gathered}
$$

This representation and formula (4.11) complete the proof of the lemma.

\section{Post-resonance expansion}

In this section we show how to connect the external asymptotic solution after the passage through the resonance with the solution in the neighborhood of the curve $l_{k}=0$.

There is a difference between these solution. The leading-order term of the solution in this section includes an additional mode. This new mode relates to the phase $\phi_{k}$ which was generated by the passage through the resonance near the curve $l_{k}=0$. It gives

$$
\Upsilon_{1,0}^{k}=\Upsilon_{1,0}^{k} \cup\left\{ \pm \phi_{k}\right\}
$$


This section consists in two parts. The first part contains the construction of the formal asymptotic solution. This solution is similar to the solution from section 3. Asymptotic behaviour for higher-order terms as $l_{k} \rightarrow 0$ follows from section 4.3.2. In the second part of this section we determine the domain of validity for this external asymptotics near resonance curve $l_{k}\left(x_{2}, t_{2}\right)=0$. The matching method gives us the initial conditions for the coefficients of the asymptotics.

5.1. Structure of the second external asymptotics. Let us construct the formal asymptotic solution of the following form with the changing of the phase set according to 5.1 .

$$
\begin{gathered}
\Psi_{1}^{k}=\sum_{m=k+1}^{N} \sum_{ \pm \phi_{m}} \exp \left\{ \pm i \phi_{m}\left(x_{2}, t_{2}\right) / \varepsilon^{2}\right\} \Psi_{1,0, \pm \phi_{m}}\left(x_{1}, t_{1}, t_{2}\right) \\
\Psi_{n}^{k}=\sum_{j=0}^{n-2} \ln ^{j}(\varepsilon) \sum_{m=k+1}^{N}\left(\sum_{ \pm \phi_{m}} \exp \left\{ \pm i \phi_{m}\left(x_{2}, t_{2}\right) / \varepsilon^{2}\right\} \Psi_{n, j, \pm \phi_{m}}\left(x_{1}, t_{1}, t_{2}\right)+\right. \\
\left.\sum_{\chi \in \Upsilon_{n, j}^{\prime \prime}} \exp \left\{i \chi\left(x_{2}, t_{2}\right) / \varepsilon^{2}\right\} \Psi_{n, j, \chi}\left(x_{1}, t_{1}, t_{2}\right)\right),
\end{gathered}
$$

where $\Upsilon_{n, j}^{k \prime}$ is a set of phase functions which is determined by

$$
\begin{aligned}
& \Upsilon_{1,0}^{k}=\left\{ \pm \phi_{k}, \pm \phi_{k+1}, \ldots, \pm \phi_{N}\right\} ; \quad \Upsilon_{2,0}^{k}=\Upsilon_{1,0}^{k} \cup\{ \pm S, \ldots, \pm N S\} \text {, } \\
& \begin{array}{c}
\Upsilon_{n, j}^{k}=\underset{n_{1}+n_{2}+n_{3}=n,}{n_{n_{1}, j_{1}}+\chi_{n_{2}, j_{2}}+\chi_{n_{3}, j_{3}}, \quad \chi_{n_{p}, j_{p}} \in \Upsilon_{n_{p}, j_{p}}^{k} .} \\
j_{1}+j_{2}+j_{3}=j
\end{array} \\
& \Upsilon_{n, j}^{k \prime}=\Upsilon_{n, j}^{k} \backslash\left\{ \pm \phi_{k}, \pm \phi_{k+1}, \ldots, \pm \phi_{N}\right\}
\end{aligned}
$$

Substitute this asymptotic solution in original equation and collect the terms of the same order with respect to $\varepsilon$. After collecting the terms with the same phase functions we obtain the recurrent system of equations for the coefficients.

The terms of the order $\varepsilon^{1}$ give us the equation $(3.2)$ for the phase function of eigen oscillations. The initial data is determined by matching condition (3.3) with the changing of indexes from $(k+1)$ to $k$.

The difference between this asymptotic constructions and constructions from section 3 consists in changing of the number of terms of the order of $\varepsilon$.

The amplitudes $\Psi_{1,0, \phi_{j}}$ are determined from

$$
\begin{array}{r}
2 i \partial_{t_{2}} \phi_{j} \partial_{t_{2}} \Psi_{1,0, \phi_{j}}+\left[\left(\partial_{t_{1}} \xi_{j}\right)^{2}-\left(\partial_{x_{1}} \xi_{j}\right)^{2}\right] \partial_{\xi_{j} \xi_{j}}^{2} \Psi_{1,0, \phi_{j}}+ \\
+i\left[\partial_{t_{2}}^{2} \phi_{j}-\partial_{x_{2}}^{2} \phi_{j}\right] \Psi_{1,0, \phi_{j}}+\gamma\left|\Psi_{1,0, \phi_{j}}\right|^{2} \Psi_{1,0, \phi_{j}}=0, \quad j=k, \ldots, N
\end{array}
$$

The higher order terms of 5.2 satisfy equation 3.9 . Here we present the following lemma which allows to match solution $(5.2)$ and solution 4.2 . 
LEMma 5.1. The asymptotics as $l_{k} \rightarrow 0$ of the solution of equation (3.9) has the form

$$
\Psi_{n, p, \phi_{k}}\left(x_{1}, t_{1}, t_{2}\right)=\sum_{j=-(n-2 p-2)}^{1} \sum_{m=0}^{-(j-1)} \Psi_{n, p, \phi_{k}}^{(j, m)}\left(x_{1}, t_{1}\right) l_{k}^{j}\left(\ln l_{k}\right)^{m}+O(1), \quad l_{k} \rightarrow 0 .
$$

Proof. Determine the order of the singularity of the right hand side of the equation as $l_{k} \rightarrow 0$. First consider equation $(3.9)$ for $n=3, p=0$. The solution of this equation gives us the coefficient $\Psi_{3,0, \phi_{k}}$. The nonlinearity contains the term $\left|\Psi_{2,0, k S}\right|^{2} \Psi_{1,0, \phi_{k}}$. The function $\Psi_{2,0, k S}$ has the singularity of the order $l_{k}^{-1}$ as $l_{k} \rightarrow 0$. It determines the order of singularity for right hand side $l_{k}^{-2}$. We construct the asymptotics of $\Psi_{3,0, \chi}$ in the form

$$
\Psi_{3,0, \phi_{k}}=\Psi_{3,0, \phi_{k}}^{-1,0} l_{k}^{-1}+\Psi_{3,0, \phi_{k}}^{0,1} \ln \left(l_{k}\right)+\Psi_{3,0, \phi_{k}}^{1,1} l_{k} \ln \left(l_{k}\right)+\widehat{\Psi}_{3,0, \phi_{k}},
$$

Substitute (5.5) in equation for $n=3$. It leads to recurrent system of equations for coefficients $\Psi_{3,0, \phi_{k}}^{(j, p)}$

$$
\begin{gathered}
-2 i \partial_{t_{2}} \phi_{k} \partial_{t_{2}} l_{k} \Psi_{3,0, \phi_{k}}^{(-1,0)}=-\Psi_{1,0, \phi_{k}}\left|\Psi_{2,0, S}\right|^{2} l_{k}^{2}, \\
2 i \partial_{t_{2}} \phi_{1} \partial_{t_{2}} l_{k} \Psi_{3,0, \phi_{k}}^{(0,1)}=L\left[\Psi_{3,0, \phi_{k}}^{(-1,0)}\right], \\
2 i \partial_{t_{2}} \phi_{k} \partial_{t_{2}} l_{k} \Psi_{3,0, \phi_{k}}^{(1,1)}=L\left[\Psi_{3,0, \phi_{k}}^{(0,1)}\right] .
\end{gathered}
$$

Here we denote the linear operator by

$$
L[\Psi]=2 i \partial_{t_{2}} \phi_{k} \partial_{t_{2}} \Psi+\partial_{\xi}^{2} \Psi+i\left[\partial_{t_{2}}^{2} \phi_{k}-\partial_{x_{2}}^{2} \phi_{k}\right] \Psi+\gamma\left(2\left|\Psi_{1,0, \phi_{k}}\right|^{2} \Psi+\left(\Psi_{1,0, \phi_{k}}\right)^{2} \Psi^{*}\right) .
$$

The regular part $\widehat{\Psi}_{3,0, \phi_{k}}$ of the asymptotics satisfies the nonhomogeneous linear Schrodinger equation. The right hand side of the equation is smooth

$$
L\left[\widehat{\Psi}_{3,0, \phi_{k}}\right]=-l_{k} \ln \left|l_{k}\right| L\left[\Psi_{3,0, \phi_{k}}^{(1,1)}\right]-2 i \partial_{t_{2}} \phi_{k} \partial_{t_{2}} l_{k} \Psi_{3,0, \phi_{k}}^{(1,1)} .
$$

The initial condition for the regular part of the asymptotics is determined below by matching with the internal asymptotic expansion.

The structure of the terms $\Psi_{n, p, \pm \phi_{k}}$ for $n>3$ has a similar form. The right hand side of equation (3.9) depends on junior terms. These singularities can be eliminate

$$
F_{n, p, \phi_{k}}=\sum_{j=0}^{-(n-2)} \sum_{m=0}^{-j+1} l_{k}^{j} \ln ^{m}\left|l_{k}\right| f_{k}^{(j, m)}+\widehat{F}_{k n, p, \phi_{1}}^{(j)} .
$$

The coefficients $f_{k_{n, p, \phi_{k}}}^{(j, m)}$ do not contain singularities as $l_{k} \rightarrow 0$. These coefficients are easy calculated.

The direct substitution of (5.4) in equation and collecting the terms with the same order of $l_{k}$ complete the proof of lemma 5.1.

5.2. The domain of validity of the second external asymptotics as $l_{k} \rightarrow+0$ and matching procedure. The domain of validity of the second external asymptotics is determined by

$$
\frac{\varepsilon \Psi_{n+1}^{k}}{\Psi_{n}^{k}} \ll 1 .
$$

Formulas 5.2 and 5.4 give the condition

$$
l_{k} \gg \varepsilon .
$$


The domain $\left|l_{k}\right| \ll 1$ of validity of the internal asymptotics and domain of validity of the second external asymptotics are intersected. This fact allows to complete the construction of the second external asymptotics by matching method 16. The structure of singular parts of the internal asymptotics as $\lambda_{k} \rightarrow+\infty$ and external asymptotics as $l_{k} \rightarrow 0$ are equivalent. The coefficients are coincided due to our constructions. The matching of regular parts of these asymptotics takes place due to

$$
\left.\Psi_{n, j, \phi_{k}}\right|_{l_{k}=0}=W_{n, j, k S}^{(0,0)}(\xi) .
$$

The function $W_{n, j, k S}^{(0,0)}(\xi)$ is determined in lemma 4.6 .

In particular, the initial condition for the amplitude $\Psi_{1,0, \phi_{k}}$ corresponding to the new generated phase $\phi_{k}$ has a form

$$
\left.\Psi_{1,0, \phi_{k}}\right|_{l_{k}=0}=\int_{-\infty}^{\infty} d \sigma f_{k}\left(x_{1}\right) \exp \left(i \int_{0}^{\sigma} d \chi \lambda_{k}\left(x_{1}, t_{1}, \varepsilon\right)\right) .
$$

The initial data for the others amplitudes $\Psi_{1,0, \phi_{j}}, \quad j=k+1, \ldots, N$ of the leadingorder term of (5.2) are represented by values of these amplitudes on the curve $l_{k}=0$.

The soliton theory for nonlinear Schrodinger equation leads us to the fact that the function $\Psi_{1,0, \phi_{j}}$ contains the solitary waves when $f_{j}\left(x_{1}\right)$ is sufficiently large.

\section{Resonances in the higher order terms of the asymptotics.}

In this section we discuss the passage through the resonances of higher order terms of the asymptotic solution only. Such passage does not change the leadingorder term and leads to changing of the solution in higher order terms only.

Equations 4.10 are solvable while the multiplier

$$
l[\chi]=\left[-\left(\chi_{t_{2}}\right)^{2}+\left(\chi_{x_{2}}\right)^{2}+1\right] \neq 0 .
$$

Let us denote a particular value of $\chi$ such that $l[\chi]=0$ by $\chi_{r}$. In the neighborhood of the curve $l\left[\chi_{r}\right]=0, \chi_{r} \in \Upsilon_{n, k}$ the coefficients of the asymptotic expansion has a form

$$
\begin{array}{rr}
\Psi_{n, k, \chi_{r}}=\mathcal{O}\left(l^{-1}\left[\chi_{r}\right]\right), & l\left[\chi_{r}\right] \rightarrow 0, \\
\Psi_{n+m, k, \chi_{r}}=\mathcal{O}\left(l^{-(m+1)}\left[\chi_{r}\right]\right), & l\left[\chi_{r}\right] \rightarrow 0 .
\end{array}
$$

These formulas give the domain of validity of $(5.2)$

$$
l\left[\chi_{r}\right] \gg \varepsilon .
$$

In the neighborhood of the curve $l\left[\chi_{r}\right]=0$ we use a new scaled variable $\lambda_{\chi_{r}}=l_{\chi_{r}} / \varepsilon$. The formal asymptotic solution is constructed in the form

$$
U(x, t, \varepsilon)=U_{n-2}(x, t, \varepsilon)+U_{\text {res }}(x, t, \varepsilon) .
$$

The asymptotic solution has two parts. The first one does not depend on the scaled variable $\lambda_{\chi_{r}}$. But the second part of the solution depends on $\lambda_{\chi_{r}}$, because the resonance on the curve $\lambda_{\chi_{r}}=0$ appears only in higher order terms of the asymptotics. The terms $U_{n-2}(x, t, \varepsilon)$ and $U_{r e s}(x, t, \varepsilon)$ have a form

$U_{n-2}(x, t, \varepsilon)=\sum_{m=1}^{n-2} \varepsilon^{m} \sum_{k=0}^{m-2} \ln ^{k}(\varepsilon)\left(\sum_{ \pm \phi} \exp \left\{ \pm i \phi\left(x_{2}, t_{2}\right) / \varepsilon^{2}\right\} \Psi_{m, k, \pm \phi}\left(x_{1}, t_{1}, t_{2}\right)+\right.$

$$
\left.\sum_{\chi_{r} \in \Upsilon_{m, k}^{\prime}} \exp \left\{i \chi_{r}\left(x_{2}, t_{2}\right) / \varepsilon^{2}\right\} \Psi_{m, k, \chi_{r}}\left(x_{1}, t_{1}, t_{2}\right)\right) \text {. }
$$




$$
\begin{array}{r}
U_{r e s}(x, t, \varepsilon)=\sum_{m=n-1}^{\infty} \varepsilon^{m} \sum_{k=0}^{m-2} \ln ^{k}(\varepsilon)\left(\sum_{ \pm \phi} \exp \left\{ \pm i \phi\left(x_{2}, t_{2}\right) / \varepsilon^{2}\right\} \Psi_{m, k, \pm \phi}\left(\lambda_{\chi_{r}}, x_{1}, t_{1}, t_{2}\right)+\right. \\
\left.\sum_{\chi_{r} \in \Upsilon_{m, k}^{\prime}} \exp \left\{i \chi_{r}\left(x_{2}, t_{2}\right) / \varepsilon^{2}\right\} \Psi_{m, k, \chi_{r}}\left(\lambda_{\chi_{r}}, x_{1}, t_{1}, t_{2}\right)\right) .
\end{array}
$$

Substitution of (6.3) into (2.1) gives the recurrent sequence of the problems for the coefficients of 6.3). Note that the coefficients $\Psi_{m, k, \pm \phi}$ and $\Psi_{m, k, \chi_{r}}$ for $m \leq(n-1)$ are determined from the standard problems as shown in section 5 .

The coefficients $\Psi_{m, k, \pm \phi}$ and $\Psi_{m, k, \chi_{r}}$ for $m \geq(n-1)$ are determined as well as the internal expansion from section 4 . Here we present the standard problems for the coefficients of the asymptotics without detailed derivation. The higher-order terms are calculated by the same way. In particularly, the terms in the case lower index is equal to $\chi_{r}$ are determined by differential equations.

$$
2 i \partial_{t_{2}} \chi_{r} \partial_{t_{1}} \Psi_{m, k, \chi_{r}}-2 i \partial_{x_{2}} \chi_{r} \partial_{x_{1}} \Psi_{m, k, \chi_{r}}-\lambda_{\chi_{r}} \Psi_{m, k, \chi_{r}}=F_{m, k, \chi_{r}} .
$$

The right hand side $F_{m, k, \chi_{r}}$ of equation (6.6) has form (3.11) with the changing of the index from $\chi$ to $\chi_{r}$.

The terms in the case the lower index is not equal to $\chi_{r}$ are determined by algebraic equations 4.10 .

The analysis of equation 6.6 is realized in section 4.2 . The passage through this resonance layer leads to a new phase function appearance as was shown in 4.3.2. We denote this new phase function by $\varphi_{r}$.

In the domain after passage through resonance layer the amplitude under this phase function is determined by

$$
\begin{array}{r}
2 i \partial_{t_{2}} \varphi_{r} \partial_{t_{2}} \Psi_{n-1, k, \varphi_{r}}+\left[\left(\partial_{t_{1}} \xi_{r}\right)^{2}-\left(\partial_{x_{1}} \xi_{r}\right)^{2}\right] \partial_{\xi_{r} \xi_{r}}^{2} \Psi_{n-1, k, \varphi_{r}}+ \\
+i\left[\partial_{t_{2}}^{2} \varphi_{r}-\partial_{x_{2}}^{2} \varphi_{r}\right] \Psi_{n-1, k, \varphi_{r}}+\gamma\left|\Psi_{1,0, \phi_{1}}\right|^{2} \Psi_{n-1, k, \varphi_{r}}=0
\end{array}
$$

here the variable $\xi_{r}$ is determined by

$$
\frac{d x_{1}}{d \xi_{r}}=\partial_{t_{2}} \chi_{r}, \quad \frac{d t_{1}}{d \xi_{r}}=\partial_{x_{2}} \chi_{r}
$$

After the passage of the resonance layer the set $\Upsilon_{n-1, k}$ is changed by the following rule

$$
\Upsilon_{n-1, k} \rightarrow \Upsilon_{n-1, k} \bigcup \chi_{r}
$$

It leads to changing of the set of phases for higher order correction terms by the ordinary way.

Acknowledgments. We are grateful to I.V. Barashenkov, L.A. Kalyakin and B.I.Suleimanov for helpful comments and for help in improving of the mathematical presentation the results.

\section{References}

[1] P.L.Kelley, Phys.Rev.Lett.,15,1005(1965).

[2] V.I.Talanov, Pis'ma v ZhETF, 2,218(1965).

[3] V.E.Zaharov, Zhurnal prikladnoi mekhaniki i tekhnicheskoi fiziki,2,86(1968).

[4] L.A. Kalyakin, Uspekhi matematicheskikh nauk,44,5(1989).

[5] L.Friedland, A.G.Shagalov, Phys.rev.lett., 8,4357(1998).

[6] S.G.Glebov, O.M. Kiselev, V.A.Lazarev. Birth of solitons during passage through local resonance. Proceedings of the Steklov Institute of Mathematics. Suppl., 2003, issue 1, S84-S90. 
[7] S.G. Glebov and O.M. Kiselev, The Stimulated Scattering of Solitons on a Resonance. Journal of Nonlinear Mathematical Physics, v.12, n3 (2005), pp.330 341.

[8] Kiselev O.M., Glebov, S. G., Lazarev V. A., Resonant pumping in nonlinear Klein-Gordon equation and solitary packets of waves. http://arxiv.org/abs/math-ph/0410041

[9] Kiselev O.M., Glebov, S. G., Lazarev V. A., Slow passage through resonance for a weakly nonlinear dispersive wave. Accepted in SIAM J.of Applied Mathematics.

[10] J. Kevorkyan, SIAM J. Appl.Math.,20,364(1971).

[11] Rubenfeld L., Stud. Appl. Math.,57,77(1977).

[12] J.C. Neu, SIAM J. Appl. Math.,43,141(1983).

[13] L.A. Kalyakin, Matematicheskie zametki,44,697(1988).

[14] S.G.Glebov, Differentsial'nye uravneniya,31,1402(1995).

[15] A. Jeffrey and T. Kawahara. Asymptotic methods in nonlinear wave theory. Pitman Publishing INC, 1982.

[16] A. M. Il'in Matching of Asymptotic Expansions of Solutions of Boundary Value Problem, AMS, 1992.

[17] V.E.Zakharov, S.V.Manakov, S.P.Novikov, L.P.Pitaevskii, Teoriya solitonov: metod obratnoi zadachi, Nauka, 1980.

InSTITUTE OF MATH. USC RAS

E-mail address: ok@ufanet.ru

Ufa State Petroleum Technical University

E-mail address: sg@anrb.ru 